\title{
Representaciones sociales del medio ambiente en estudiantes de educación media y superior de la ciudad de Santa Marta, Colombia*
}

\section{Social representations about the environment in middle and high school students in the city of Santa Marta, Colombia}

\author{
Ubaldo Rodríguez de Ávila* \\ Carmelina Paba Barbosa \\ Zuany Luz Paba Argote \\ Kelly Obispo Salazar \\ Sharol Cortés Miranda \\ Universidad del Magdalena, Colombia \\ Recibido: 26 de octubre de 2018 \\ Revisado: 6 de diciembre de 2018 \\ Aceptado: 4 de enero de 2019
}

\section{Resumen}

El presente artículo describe el contenido, la organización y el significado de la representación social del medio ambiente, admitiendo que un fenómeno permite diversas interpretaciones, desde el paradigma epistemológico de los enfoques interpretativos, de tipo cualitativo, con técnicas de procesamiento de la información de tipo cuantitativo. Participaron 280 estudiantes de educación media y superior de la ciudad de Santa Marta, Colombia, 54\% mujeres y $46 \%$ hombres. Se hizo un análisis prototípico y lexicográfico de la representación social (RS), basado en la técnica de análisis de contenido y la teoría del discurso. Se encontraron un total de 1684 palabras o expresiones evocadas, de las cuales 194 fueron palabras diferentes (1 palabra diferente por persona), además se lograron identificar varias categorías para comprender la organización y estructura del significado de la RS del Medio Ambiente. Se discuten los resultados a la luz de la teoría de las Representaciones Sociales, en la medida que no se evidencia heterogeneidad en la representación social del medio ambiente.

Palabras clave: representación social, medio ambiente, análisis de contenido.

Artículo de investigación. Cómo citar: Rodríguez, A. U., Paba, B. C., Paba, A. Z. L., Obispo, S. K., \& Cortés, M. S. (2019). Representaciones sociales del medio ambiente en estudiantes de educación media y superior de la ciudad de Santa Marta, Colombia. Diversitas: Perspectivas en Psicología, 15(2), 301-314. DOI: 10.15332/22563067.3678

** Correspondencia: Ubaldo Rodríguez de Ávila. Facultad de Psicología. Universidad de Magdalena, Santa Marta, Colombia. Cll. 32 n. ${ }^{\circ} 22-08$. Correo electrónico: rodriguez.ubaldo@gmail.com 


\section{Abstract}

The present article aims at describing the content, organization and meaning of the Social Representation of the environment, admitting from its point of view that a phenomenon allows for different interpretations, from the epistemological paradigm of interpretative approaches, of qualitative type, with processing techniques quantitative information. 280 students of middle and higher education in the city of Santa Marta, Colombia participated, 54\% women $46 \%$ men. A prototypical and lexicographic analysis of the Social Representation (SR) was made, based on the technique of content analysis and discourse theory. A total of 1684 words or evoked expressions were found, of which 194 are different words (1 different word per person), and several categories were identified to understand the organization and structure of the meaning of SR of the Environment. The results are discussed considering the theory of Social Representations to the extent that there is no evidence of heterogeneity in the Social Representation of the Environment.

Keywords: Social Representation, environment, content analysis.

\section{Introducción}

Machado (1997) informa que nada parece ser más difícil que cambiar los modos de comportamiento de una sociedad cuando el estilo de desarrollo imperante está muy arraigado. Sin embargo, los grandes pedagogos y educadores de la historia concuerdan que en la educación está la base de la transformación social, y en este sentido, en la solución de los problemas ambientales; de esta forma y por extensión, en la educación ambiental está el deber de dicha transformación. Transformación en valores, símbolos, ideologías de la existencia, hacia una actitud transformativa y de renovación.

Ahora bien, en un informe de un grupo de más de 2500 científicos organizado por Naciones Unidas aumenta el grado de conocimiento sobre el cambio climático como producto de la crisis ambiental global y da cuenta de la responsabilidad del hombre en la proliferación del fenómeno (Laría, 2007). En este sentido, como expresa Capalbo (2005) si nos preguntáramos acerca de la magnitud y alcance de la crisis ambiental, podríamos proponer tres categorías conceptuales para enmarcar una posible respuesta: a) La crisis es perturbadora para la vida, empeorando las condiciones de amplias poblaciones, pero sin amenazar la continuidad de la especie humana en su conjunto, b) La crisis perturba pro- fundamente el ecosistema planetario, el cual será capaz, sin embargo, de restablecer su homeostasis, aunque su nuevo estado de equilibrio, excluirá a la especie humana. c) La crisis acabará destruyendo integralmente el ecosistema planetario, pasando la Tierra a ser un planeta desierto, desprovisto de vida, tal como se la define hoy.

Los problemas ambientales se encuentran relacionados unos con otros en una interdependencia trascendente para la existencia humana. Hoy en día nos enfrentamos a una auténtica crisis ambiental de carácter global en una multiplicidad de fenómenos que constituyen síntomas de lo que se denomina “crisis ambiental” (Crespo \& Garcés, 2002). Esta multiplicidad de fenómenos, tal como consideran Crespo y Garcés (2002, p. 2), encierra diversos aspectos tales como emisiones de $\mathrm{CO}_{2}$ en la atmosfera, aumentando la temperatura global y afectando el equilibrio térmico del planeta y sus patrones climatológicos; disminución de la biodiversidad o desaparición de especies, que afectan los procesos económicos y sociales de la especie humana y su desarrollo evolutivo. Así mismo, se presenta una disminución de la superficie arbolada, a través de la tala de bosques y la quema de estos ocasionando la desertización del suelo; igualmente el ciclo hidrológico, la regulación genética, la vida social de los hombres y otros aspectos relacionados. 
A partir de la década de los años setenta, en el ámbito nacional ha crecido cada vez la preocupación por encontrar soluciones a la crisis ambiental, y para esto se planteó la educación ambiental como una de las estrategias importantes; es así que en Colombia se vienen desarrollando acciones que buscan la inclusión de la dimensión ambiental como uno de los componentes fundamentales del currículo de la formación formal y de las actividades de la educación no formal e informal (Ministerio del Medio Ambiente, 2002).

Actualmente, el sistema educativo colombiano proyecta estrategias de renovación que buscan amoldarse a las nuevas exigencias tecnológicas y medio ambientales, concebido el ambiente como un compendio de factores naturales, sociales y culturales que interaccionan con la vida del hombre en sus dimensiones material y psicológica (Navarro, 2005; Rodríguez, 2006; Rodríguez, Cortés \& Varela, 2007).

La perspectiva de análisis del problema del ambiente a través de las representaciones sociales será la consideración de ella misma como un producto de decisiones sociales (culturales, de desarrollo productivo, políticas y sociológicas e institucionales). De igual forma, como lo manifiesta Tilbury (2004) en lo referente al problema ecológico y ambiental, la posibilidad real de su solución por los mismos medios: la modificación de los patrones e indicadores del "desarrollo social" y la racionalidad de tipo reflexiva por parte de las organizaciones humanas que permita dar claridad a las sociedades que, con el actual despliegue técnico logrado y la riqueza producto de la transformación del medio, es posible mantener en óptimas condiciones de vida a más del doble de la cifra actual de habitantes del planeta. Es decir, conocer las representaciones sociales sobre el medio ambiente y la educación ambiental permitirá identificar las formas comportamentales de los sujetos, y así incidir mejor para que emerjan conductas proambientales.

En este artículo se describe la representación social del medio ambiente y la educación ambiental en estudiantes de educación media y superior de la ciudad de Santa Marta, Colombia, cuyos objetivos específicos están centrados en: la identificación del contenido, la descripción de la organización y la identificación el significado de la representación social del medio ambiente.

\section{Representación social}

La Real Academia de la Lengua Española (2004) define la palabra "representación" como "acción de representar", y ésta última palabra la conceptualiza como "presentar de nuevo", "hacer las veces de otro", "figura”, "imagen”, "cosa que expresa otra". Y la palabra "social" se entiende como lo "relativo a la sociedad", del Latín societas, estado de los hombres que viven bajo leyes comunes; de esta forma, toda imagen, figura o cosa que expresa otra, guiada bajo leyes que se dan entre los sujetos de forma común, es lo que se concibe como representación social (Rodríguez et al., 2007).

Como lo expresa Navarro (2013) las representaciones sociales son formas de pensamiento social, socialmente construidas $y$, en consecuencia, con una utilidad práctica. En lo que concierne al ámbito ambiental, esta teoría ha demostrado un interés mayor en la comprensión de la base sociocognitiva subyacente a diferentes comportamientos y tomas de posición frente a este objeto de interés social (Navarro, 2013). En este sentido, dicho concepto relaciona varios aspectos que acercan a la comprensión de la realidad de las otras personas, como la experiencia, el sistema cultural en el que se desenvuelven, la sociedad y el grupo social con el que se relacionan.

Desde la perspectiva sociológica y psicosocial, se destaca el papel de la influencia de la sociedad sobre el conocimiento social. Por ejemplo, algunos autores afirman que las representaciones sociales tienen su origen en las representaciones colectivas (Ceirano, 2000; Durkheim, 1996). Por su parte, Jodelet (1984) señala que las representaciones sociales constituyen modalidades de pensamiento práctico orientadas hacia la comunicación, la comprensión y el dominio del entorno social, material e ideal. Se parte entonces desde las representaciones sociales como teoría psicológica, en primer lugar, de las observaciones hechas por Berger y Luckmann (1988), Schutz y Luckmann (1977) y Ceirano (2000), de la noción de la no existencia de 
un mundo "verdadero", pre-existente a los fenómenos. Esta noción operaría como el fundamento de toda explicación y desde el cual se presupondrían significaciones y la existencia de distintas construcciones sociales de la realidad. Esto se evidencia en el sentido de que cada grupo es co-constructor de una idea de la realidad en la experiencia vivida y compartida, una idea de verdad, una idea de error y una idea de normalidad, como dice Lahitte, Hurrel y Malpartida (1989).

\section{Representación Social y Medio Ambiente}

En el concepto de Rodríguez, Cortés y Varela (2007) el medio ambiente debe entenderse como un compendio de aspectos naturales, sociales y culturales que afectan la vida material y psicológica del hombre. Son muchos los significados que se pueden extraer del término, sin embargo, la definición anterior es la que más se ajusta a la manera ecológica de interpretación psicológica.

Lo ambiental se entiende como un espacio interdisciplinario, donde confluyen las diferentes especialidades en un diálogo de saberes que trata de aportar, desde una perspectiva compleja, elementos de análisis que se creen útiles y necesarios ante el evidente fracaso de las ciencias tradicionales para resolver algunos problemas básicos de la sociedad como pobreza, violencia y deterioro del entorno (Suárez \& Márquez, 2004).

Suárez y Márquez (2004) consideran lo ambiental también como lo relativo al estudio de las relaciones de la sociedad con los ecosistemas y sus recursos. Estas relaciones son el núcleo de lo "ambiental", aunque lo que pueda entenderse por "ambiental" siga en discusión. Mientras Flores (2010) dice que "el medio ambiente es un objeto social complejo, cultural y contextualmente determinado. Es socialmente construido, por lo que escapa a cualquier definición precisa, global y consensual. El medio ambiente es un producto social, que refleja el sentido que los estudiantes le proporcionan en un momento específico" (p. 411).

Los científicos desde las ciencias naturales tienden a identificar ambiente con medio o entorno biofísico, enfoque calificado como reduccionista, pues tiende a marginar la cuestión humana, fundamental en procesos que no sólo la ciencia, sino el uso común, llaman ambientales (deterioro ambiental, impacto ambiental), e implican a la sociedad en interacción con el resto de la naturaleza. De allí una confusión frecuente entre ambientalismo y ecología, la rama de la biología que estudia la estructura y funcionamiento de los sistemas ecológicos (bosques, mares, ríos y sus componentes), una área de fundamental importancia para ayudar a comprender las relaciones de la sociedad con su entorno pero que no tiene como objeto en sí su estudio (Suárez \& Márquez, 2004).

Se entiende entonces por medio ambiente al entorno que afecta y condiciona especialmente las circunstancias de vida de las personas o la sociedad en su conjunto. Comprende el conjunto de valores naturales, sociales y culturales existentes en un lugar y un momento determinado, que influyen en la vida humana y las generaciones venideras. Es decir, no se trata sólo del espacio en el que se desarrolla la vida, sino que también abarca seres vivos, objetos, agua, suelo, aire y las relaciones entre ellos, así como elementos intangibles como la cultura.

Ferreira (2002) cita una investigación de Reigota (1995) la cual ofrece reflexiones teóricas de los estudios con representaciones sociales sobre medio ambiente y expone los resultados de una investigación realizada en 1991 con maestros que cursaban la cátedra "Fundamentos y tendencias de la educación ambiental", en la Facultad de Filosofía, Ciencias y Letras, de Gurapuava, en el Estado de Paraná, orientada a identificar las representaciones sociales y las prácticas pedagógicas de cada uno, relacionadas con el tema medio ambiente. En $A$ Floresta e a Escola (Reigota, 199, citado en Ferreira, 2002), además de las aportaciones teóricas, ofrece una propuesta pedagógica basada en el estudio y la interpretación de imágenes (dibujos, caricaturas, fotografías, cubiertas de libros, publicidad, entre otros), considerando que aquéllas originan, difunden y materializan representaciones sociales presentes en los medios profesionales, culturales, sociales y académicos, solidificando preconceptos y estereotipos, al tiempo que legitiman posiciones equivocadas. 
El tema del medio ambiente viene siendo tratado ampliamente desde hace cuarenta años. Esto se constata al indagar los antecedentes de la Conferencia de Estocolmo en el año 1972, del Seminario de Belgrado en 1975, de la Conferencia de Nairobi en 1976, de la Reunión de Tbilisi en 1977, del Encuentro de Moscú en 1978, de la conferencia de Malta en 1991, del Seminario de El Cairo 1991, de Acción 21 en 1992, de la Conferencia de Rio en 1992, del Encuentro de Chile en 1995, del Encuentro de Cuba en 1995, del Encuentro de Paraguay en 1995, de la Conferencia Internacional de la UICN, en Montreal en 1996, de la Reunión Iberoamericana de Educación Ambiental para los Países de la Región Central de América Latina, Managua, en 1996, de la Reunión Iberoamericana de Educación Ambiental para los Países del Sur de América Latina, Bogotá, en 1997, de la Reunión Iberoamericana de Educación Ambiental para los países del Cono Sur, Argentina, en 1997, de la Conferencia Internacional sobre Ambiente y Sociedad: Educación y consciencia pública para la sustentabilidad en 1997, del II congreso Iberoamericano de Educación Ambiental, México, en 1998, del III Congreso Iberoamericano de Educación Ambiental, Venezuela, en el año 2000, de la Reunión Internacional de Expertos en educación Ambiental, España, en el año 2000, entre otros.

De otra parte, Palavecinos et al. (2008), hablan de los comportamientos proambientales en un estudio del comportamiento de queja ciudadana, implementando un sistema participativo de gestión ambiental en Madrid. También encontramos a Meira (2008, citado en Rodríguez \& López, 2005) tratando los problemas ambientales globales y educación ambiental, en una aproximación desde las representaciones sociales del cambio climático.

Por su parte, Rodríguez y López (2005) describen las representaciones sociales de conservación ambiental en la comunidad la Flor de Marqués de Comillas, Chiapas. Y Ferreira (2002) describe las representaciones sociales de medio ambiente y educación ambiental de docentes universitarios. Al mismo tiempo, González (1998) describe en su estudio la relación de la educación ambiental y la formación del profesorado y Torres (1998), al estudiar la educación ambiental, propone una estrategia flexible, un proceso y unos propósitos en permanente construcción en educación ambiental en Colombia.

En las diversas investigaciones analizadas, sobre las representaciones del medio ambiente en ciudades de Centro y Suramérica, Flores (2013) aclara que se evidencia su importancia para comprender la práctica de la educación ambiental. De esto se deduce que para la educación ambiental resulta fundamental comprender las formas en que se establecen las representaciones sociales, en la desconstrucciónconstrucción de los elementos que forman su pensamiento cotidiano (Flores, 2013). Por todo eso Flores (2013) expresa que a partir de los resultados de las investigaciones revisadas, se puede afirmar que el medio ambiente es un objeto social complejo, cultural y contextualmente determinado.

\section{Método}

\section{Diseño}

La presente investigación pretende la descripción de la representación social del medio ambiente, asumiendo diversas interpretaciones; esta perspectiva representa el punto de partida del abordaje investigativo, el cual se fundamenta sobre el paradigma epistemológico de los enfoques interpretativos, de tipo cualitativo. Sin embargo, también se aborda el tema desde un procedimiento cuantitativo. Se pretende una integración de dos perspectivas analíticas para dar cuenta de los resultados, con influencia del enfoque crítico social en la medida que la temática del ambiente exige un direccionamiento crítico en el sentido social de los resultados.

La propuesta metodológica utilizada en esta investigación parte del reconocimiento explícito de la complejidad del objeto de estudio. Esta complejidad que es elaborada teóricamente en el marco conceptual, necesariamente conlleva a abordar metodológicamente la investigación a partir de la perspectiva de análisis en el Marco de lo Social, donde la misma tiene muchos propósitos, uno de los cuales es la descripción (Babbie, 2000, p. 72); pues, desde la perspectiva de éste autor, "la descripción es un objetivo de la investigación social”, por tanto, el tipo de investigación es descriptivo. 


\section{Población y Muestra}

Participaron 280 sujetos de los cuales el $66 \%$ corresponden a sujetos en edades de 16 a 20 años de edad; le sigue el rango de 21 a 25 años de edad con un porcentaje de $18 \%$, seguido del rango de edad de 11 a 15 años (13\%) el restante $3 \%$ se encuentran ubicados en el rango de 26 años o más. El 54\% de los sujetos pertenecen al género femenino y en un $46 \%$ al género masculino.

\section{Instrumentos y Técnicas}

En cuanto al instrumento, éste fue diseñado bajo la técnica de asociación libre de palabras, evocadas a partir de la palabra inductora "Medio Ambiente". En este sentido solo se necesita una frase que invita a declarar todas las palabras o expresiones que a los entrevistados se les ocurra cuando piensan en esta palabra inductora. Pues la hipótesis de partida de esta técnica de recolección de información es la existencia de un funcionamiento cognitivo a partir del cual "algunos términos son inmediatamente movilizados para expresar una representación" (Vérges 1994, p.235, citado en Navarro, 2004, 2006, 2010). En efecto, el carácter espontáneo y proyectivo de esta técnica permite acceder a los elementos que hacen parte del universo semántico del objeto estudiado (Abric 1994, citado en Navarro, 2005).

Por otro lado, el tratamiento de los datos y su interpretación estuvo dado a través del abordaje del análisis de contenido, previamente elaborado por la Teoría del Discurso para la comprensión de las representaciones sociales. Además de esto, se utilizó el programa estadístico SPSS versión 15.0 para procesar la información referente a las variables de los sujetos e ítems.

\section{Resultados}

En cuanto al contenido de la representación social del medio ambiente que los estudiantes de la Universidad del Magdalena tienen construido se encontró que se produjeron 1684 palabras o expresiones ( 6 expresiones por sujeto) de las cuales 194 fueron palabras diferentes, es decir, un promedio de 1 palabra diferente por persona.
Para entender la organización de la representación social del medio ambiente se realizó un análisis del prototipo, según la técnica Vergés (Navarro et al., 2005), con el fin de reconocer la jerarquía de los elementos que la componen y su organización estructural, permitiendo comprender el sentido particular que para los sujetos sociales tienen de la RS del Medio Ambiente. Se extrae la co-ocurrencia de las palabras más significativas de las evocaciones de los sujetos, que corresponden al $20 \%$ de las evocaciones totales (ver tabla 1), ordenadas alfabéticamente.

Se obtuvo luego el análisis de prototipicidad, un segundo corpus de 38 palabras (ver tabla 2), las más importantes para referirse al objeto de representación (este abordaje permite determinar la organización de la representación social del medio ambiente [RS del MA]).

En primer lugar, se descubrió que en el núcleo central, Casilla uno se sitúan las palabras más evocadas (fuerte frecuencia) y enunciadas en primer lugar (rango de aparición). Luego se obtuvo el análisis de prototipicidad, un segundo corpus de 38 palabras (ver tabla 2), las más importantes para referirse al objeto de representación. De esta forma, en la casilla uno se sitúan las palabras más evocadas, lo cual indica que estos son los elementos hipotéticamente centrales, es decir que hacen parte del núcleo central de la Representación Social del Medio Ambiente.

En la Casilla dos se ubican las palabras de frecuencia y rango débil, y en la tres se sitúan los términos de rango y frecuencia fuerte, los cuales constituyen la zona ambigua de la representación, estos elementos son periféricos pero tienen una alta probabilidad de convertirse en centrales. Por último, en la casilla 4 se sitúan los elementos periféricos de la representación, con una frecuencia débil y un rango de aparición fuerte. No obstante, se observa en la tabla que esta casilla está vacía. A partir de esta interpretación útil y necesaria, un análisis de categorías es propuesto para conocer los temas que componen la RS del MA y que ayudan a identificar además del Contenido, toda la Organización de la RS del MA. Cinco categorías surgieron inicialmente del análisis prototípico (ver tabla 3) organizando el sentido de las 38 palabras del prototipo. 
Tabla 1.

Coocurrencias de palabras evocadas

\begin{tabular}{|c|c|c|c|}
\hline Palabra & Frecuencia & $\%$ & Rango \\
\hline Naturaleza & 292 & 17.34 & 786 \\
\hline Seres vivos & 206 & 12.23 & 866 \\
\hline Cuidado de la naturaleza & 70 & 4.16 & 176.1 \\
\hline Entorno & 65 & 3.86 & 218.1 \\
\hline Aire & 58 & 3.44 & 188.1 \\
\hline Vida/vivir & 58 & 3.44 & 207.1 \\
\hline Planeta tierra/tierra & 56 & 3.33 & 212.1 \\
\hline Ecosistemas acuáticos & 49 & 2.91 & 195.1 \\
\hline Contaminación/crisis ambiental & 48 & 2.85 & 181.1 \\
\hline Árboles & 42 & 2.49 & 116.2 \\
\hline Agua & 36 & 2.14 & 137.2 \\
\hline Ecosistema & 35 & 2.08 & 111.2 \\
\hline Limpieza & 26 & 1.54 & 81.3 \\
\hline Lugar donde viven seres humanos y animales & 24 & 1.43 & 72.3 \\
\hline Vegetación & 23 & 1.37 & 89.3 \\
\hline Capa de ozono & 22 & 1.31 & 57.3 \\
\hline Atmosfera & 18 & 1.07 & 56.3 \\
\hline Cultura & 18 & 1.07 & 88.4 \\
\hline Protección/proteger & 18 & 1.07 & 57.4 \\
\hline Salud/saludable & 18 & 1.07 & 65.4 \\
\hline Calentamiento & 17 & 1.01 & 54.4 \\
\hline Oxígeno & 17 & 1.01 & 76.4 \\
\hline No arrojar basura & 13 & 0.77 & 31.5 \\
\hline Reciclaje & 15 & 0.89 & 40.5 \\
\hline Ecología & 14 & 0.83 & 42.5 \\
\hline Paisajes & 13 & 0.77 & 38.5 \\
\hline Basura & 12 & 0.71 & 42.6 \\
\hline Tranquilidad & 12 & 0.71 & 48.6 \\
\hline Deforestación & 11 & 0.65 & 33.6 \\
\hline Deterioro & 11 & 0.65 & 24.4 \\
\hline Recursos naturales & 11 & 0.65 & 43.6 \\
\hline Ciudad & 10 & 0.59 & 30.7 \\
\hline Lugar agradable & 10 & 0.59 & 26.6 \\
\hline Espacio & 9 & 0.53 & 22.7 \\
\hline Medio donde vivimos & 9 & 0.53 & 19.7 \\
\hline No contaminación & 9 & 0.53 & 26.8 \\
\hline Paz & 8 & 0.48 & 33.8 \\
\hline Sociedad & 8 & 0.48 & 27.9 \\
\hline Total & 1684 & 100 & \\
\hline Promedio & 17.3 & 0.50 & 121.8 \\
\hline
\end{tabular}

Fuente: elaboración propia. 
Tabla 2.

Análisis prototípico (Rango X Frecuencia) de la RS del MA

\begin{tabular}{|c|c|c|c|}
\hline & & FRECUEN & $\mathrm{ClA}$ \\
\hline & & FUERTE > 17.3 & DÉBIL < 17.3 \\
\hline & & Casilla 1 & Casilla 2 \\
\hline $\begin{array}{l}\text { O } \\
\underset{\alpha}{Z}\end{array}$ & 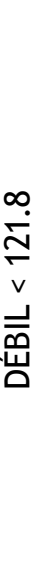 & $\begin{array}{l}\text { ARBOLES } \\
\text { AGUA } \\
\text { ECOSISTEMA } \\
\text { LIMPIEZA } \\
\text { LUGAR DONDE VIVEN SERES HUMANOS Y ANIMALES } \\
\text { VEGETACIÓN } \\
\text { CAPA DE OZONO } \\
\text { AFMOSFERA } \\
\text { CULTURA } \\
\text { PROTECCIÓN/PROTEGER } \\
\text { SALUD/SALUDABLE } \\
\text { CALENTAMIENTO } \\
\text { OXIGENO }\end{array}$ & $\begin{array}{l}\text { CALENTAMIENTO } \\
\text { OXÍGENO } \\
\text { NO ARROJAR BASURA } \\
\text { RECICLAJE } \\
\text { ECOLOGÍA } \\
\text { PAISAJES } \\
\text { BASURA } \\
\text { TRANQUILIDAD } \\
\text { DAÑO/DESASTRES/DEFORESTACIÓN } \\
\text { DETERIORO } \\
\text { RECURSOS NATURALES } \\
\text { CIUDAD } \\
\text { LUGAR AGRADABLE } \\
\text { ESPACIO } \\
\text { MEDIO DONDE VIVIMOS } \\
\text { NO CONTAMINACIÓN } \\
\text { PAZ } \\
\text { SOCIEDAD }\end{array}$ \\
\hline & 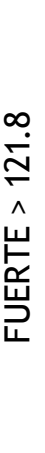 & $\begin{array}{l}\text { NATURALEZA/FLORA/FAUNA/BOSQUES } \\
\text { SERES VIVOS/SERES HUMANOS/NIMALES } \\
\text { CUIDADO DE LA NATURALEZA } \\
\text { ENTORNO/TODO LO QUE TENEMOS } \\
\text { RODEA/CONTEXTO/MEDIO QUE NOS RODEA } \\
\text { AIRE } \\
\text { VIDA/VIVIR } \\
\text { PLANETA TIERRA/TIERRA } \\
\text { ACOSISTEMAS ACUATICOS } \\
\text { HUMEDALES/LAGOS/MANGLARES/MARES/RIOS/OCEANOS } \\
\text { CONTAMINACIÓN/CRISIS AMBIENTAL } \\
\text { ÁRBOLES } \\
\text { AGUA } \\
\text { ACOSISTEMA }\end{array}$ & \\
\hline
\end{tabular}

Fuente: elaboración propia.

Tabla 3.

Categorías presentes en el Prototipo de la RS del MA.

\begin{tabular}{lllll}
\hline Naturaleza & Contaminación & Vida & Contexto & Cuidado/armonía \\
\hline $\begin{array}{l}\text { Árboles } \\
\text { Ecología }\end{array}$ & Basura & Aire & Ciudad & Cuidado de la naturaleza \\
$\begin{array}{l}\text { Ecosistemas } \\
\text { acuáticos }\end{array}$ & Contaminación & Atmósfera & Entorno espacio & Limpieza \\
Naturaleza & Deforestación & Capa de ozono & $\begin{array}{l}\text { Lugar donde viven seres } \\
\text { humnos y animales }\end{array}$ & Reciclaje \\
$\begin{array}{l}\text { Paisajes } \\
\text { Recursos }\end{array}$ & Deterioro & Oxígeno & Medio donde vivimos & Tranquilidad \\
Seres vivos & & Salud & Planeta tierra & Protección \\
Vegetación & & Vida & Sociedad & Paz \\
& & & & No contaminación \\
\hline
\end{tabular}

Fuente: elaboración propia. 
Tabla 4.

Significado de Medio Ambiente

\begin{tabular}{|c|c|c|c|c|}
\hline Categorías & Subcategorías & Códigos & $\begin{array}{l}\text { Ocurrencias (No. De } \\
\text { Evocaciones) }\end{array}$ & $\begin{array}{l}\% \text { de Ocurren- } \\
\text { cias }\end{array}$ \\
\hline \multirow{3}{*}{ Vida } & Vida & Salud & 30 & 13 \\
\hline & \multirow{2}{*}{ Creación De Dios } & Vida & 14 & 6 \\
\hline & & Creación de Dios & 1 & 0.44 \\
\hline Ecosistema & Naturaleza & Naturaleza & 68 & 30 \\
\hline Espacio higiénico & Higiene & Espacio higiénico & 12 & 5 \\
\hline \multirow{3}{*}{ Planeta tierra } & \multirow{2}{*}{ Medio que nos rodea } & Entorno & 85 & 37 \\
\hline & & Hogar & 5 & 2. 20 \\
\hline & Entorno armónico & Medio relajante & 4 & 2 \\
\hline Problema & & & 3 & 1.32 \\
\hline Responsabilidad & & & 3 & 1.32 \\
\hline Sociedad actual & & & 2 & 0.88 \\
\hline Total & & & 227 & 100 \\
\hline
\end{tabular}

Fuente: elaboración propia.

Respecto al significado del medio ambiente se obtuvieron siete (7) categorías: Vida, ecosistema, espacio higiénico, planeta tierra, problema, responsabilidad, sociedad actual (ver tabla 4)

En la tabla 4 se observa que la categoría con mayor número de ocurrencias o evocaciones de palabras y frases fue "Planeta tierra", con 106 palabras y un porcentaje del $47 \%$; seguida de la categoría "Ecosistema" con 68 palabras y con el $30 \%$; en la categoría "Vida" 45 palabras fueron evocadas, con un porcentaje del 20\%; en las categorías "Problema" y "Responsabilidad" el número de palabras fue de 3 y con un porcentaje cada una de $1.32 \%$; y por último, en la categoría "Sociedad Actual" 2 palabras fueron evocadas, presentando un porcentaje del $0.88 \%$.

En la categoría “Planeta Tierra" los participantes dieron respuestas tales como: entorno, hábitat y hogar; de la misma manera en la categoría "Ecosistema" se encontraron expresiones como Naturaleza y Fauna y Flora; y por otro lado, para referirse a la categoría "vida" los sujetos respondieron: creación de Dios, salud, cuidado y vida.

\section{Discusión}

En el abordaje de las representaciones sociales se diferencian los elementos significativos y estables (núcleo central), de aquellos que son cambiantes (sistema periférico) y que incluyen las características individuales en función del contexto en que se desempeña el sujeto (Flores, 2008). Así, conforme a los resultados obtenidos observamos que el núcleo central de la representación social del medio ambiente está compuesto por varios factores que permiten identificar la construcción de la realidad de los participantes, en relación con el medio ambiente. Se observa entonces, que para los participantes el medio ambiente puede ser visto desde diferentes perspectivas, en las que confluyen factores naturales, sociales, culturales y espaciales, elementos que rodean a los seres vivos, y que son fuente vida. Esto coincide con la definición de medio ambiente propuesta por Rodríguez et al (2007), la cual debe ser considerada como un compendio de factores naturales, sociales y materiales que afectan la vida física y psicológica del hombre, lo que permite identificar en el medio ambiente un conjunto de aspectos que hacen referencia no solo a lo natural, también a lo construido, a la cultura, a lo físico.

Por otra parte, los participantes expresaron que el medio ambiente es el "lugar donde vivimos los seres humanos y los animales, la capa de ozono, atmosfera y oxígeno", elementos que hacen parte del factor espacial y permiten percibir la composición 
que ellos dan del entorno en donde los seres vivos se desenvuelven. Vale la pena destacar que en la definición del medio ambiente se integra también la cultura, aquello que el ser humano construye y que hace parte de sus creencias y costumbres, las cuales van a conducir las acciones frente al contexto en el que se encuentra el individuo. Y como expresa Navarro (2013) las representaciones sociales son formas de pensamiento social, socialmente construidas $y$, en consecuencia, con una utilidad práctica (Navarro, 2013). Todo esto coincide con la distribución de referencia del medio ambiente, ya que para el $91 \%$ de los participantes el medio ambiente se refiere a lo natural, no obstante para el $90 \%$ la cultura no está relacionada con él, lo que significa que es ajeno a las costumbres y pensamientos propios de un grupo de personas, el MA es externo, es la naturaleza pero no incluye las creencias, pensamientos y costumbres compartidas por una sociedad.

De acuerdo con los resultados obtenidos, no se evidencia heterogeneidad en la representación social del medio ambiente, ya que no se encontró, por medio del análisis prototípico, el sistema periférico, que permite la evolución y cambio de dicha representación, esto a través de las características individuales y el contexto en que se desenvuelve el individuo. El sistema periférico tiende a preservar al núcleo de posibles transformaciones, es flexible y variable debido a que es más sensible al contexto inmediato (Flores, 2008). Significa que la información obtenida es homogénea y que la mayoría de los participantes comparten las creencias, opiniones y conocimientos sobre el medio ambiente. Es así como el núcleo central de la representación social del medio ambiente de los participantes es más resistente al cambio y poco sensible al contexto inmediato (Ruíz \& Coy, 2004). No obstante, se desconocen los elementos que permiten adaptar al núcleo central, en una situación concreta, ya que no se evidenció el sistema periférico que funciona como mecanismo de defensa, de dicho núcleo, es decir, no se observaron elementos que le permitan a la representación mantener un contacto con la realidad y el núcleo central.

Los resultados obtenidos, gracias al análisis prototípico, permiten reconocer la homogeneidad existen- te en la representación social del medio ambiente, lo cual quiere decir que para los participantes el medio ambiente incluye un conjunto de factores naturales, sociales, culturales y espaciales que hacen parte del contexto del ser humano y que lo proveen de vida. De la misma manera, a través del corpus inicial del contenido de la representación social del medio ambiente se logra confirmar la homogeneidad de la información obtenida, lo cual permite determinar que la información hallada en esta investigación, en relación con el medio ambiente, es socialmente compartida. Para ellos, factores como la naturaleza, la sociedad, la cultura y el espacio integran el medio ambiente y lo definen incluyendo en ellos los fenómenos que lo afectan.

Desde la propuesta de Duveen (2013) quien se basa en la lógica de Moscovici (2013) la Teoría de las representaciones sociales crean un puente entre los conceptos sociológicos y los psicológicos, el cual "buscó tanto reconocer un fenómeno social específico, como proporcionar los medios para hacerlo inteligible como un proceso sociopsicológico" (Duveen, 2013, p.28). Como puede verificarse en el presente estudio, a partir de la vivencia particular de los sujetos en un medio ambiente específico, en la teoría de las representaciones sociales no puede aislarse a la persona del contexto, principalmente desde el enfoque fundamental que comparten todas las Ciencias Sociales, de las cuales se desprende la idea de que todo conocimiento es socialmente compartido (Gómez et al., 2011; Rodríguez, 2006; 2009; 2011; Rodríguez et al., 2006; Rodríguez, Espitia, Montenegro, Ortiz \& Suárez, 2013; Rodríguez \& Paba, 2013). De esta forma, la representación social es un medio para socializar conceptos, pues se entiende que "la representación social se centra en la comunidad, las prácticas colectivas y la institucionalización del conocimiento social” (Farah, 2011, p. 1593).

Así, de acuerdo con Touraine (2007) sobre el "discours interprétatif dominant", dislocado en investigación hacia lugares exteriores al sujeto mismo en el análisis e interpretación de fenómenos sociales y de las conductas humanas sociales, se ha reintroducido una dimensión social en el abordaje de los fenómenos estudiados y al mismo tiempo se ha eliminado la idea de sujeto como entidad psicológica y mental, "volviendo la atención únicamente a los 
fenómenos de interacción y excluyendo todo un espacio ligado a la dinámica psíquica en la que se apoya la producción del pensamiento y de la acción" (Jodelet, 2015, p 318), crítica fundamental a los métodos positivistas (Jodelet, 2011; Moscovici, 1981, 1984, 1990, 1998, 2013; Silva Carmo, \& Silva, 2015).

Cuando se analiza el continente semántico de las representaciones sociales del medio ambiente, se encuentra que el conocimiento es socialmente compartido, y puede ser tratado desde múltiples posibilidades, desde las nociones filosóficas de aprendizaje hasta los relatos de la Epistemología Social, en el que se presenta la "cognición socializada" (Knight, 2013; Knight, Arastoopour, Williamson, Buckingham \& Littleton, 2014; Knight \& Littleton, 2015) o cognición epistémica que es "socializada", poniendo como fundamento la naturaleza normativa y pragmática de las reivindicaciones de conocimiento, el contexto social en el que las demandas de saber ocurren a un nivel macro, incluyendo el contexto disciplinario y cultural, igualmente el contexto comunicativo en que se producen tales afirmaciones, las formas en que los individuos y los grupos pequeños expresan y construyen sus afirmaciones sobre el conocimiento.

Falcon en el año 2000, según comentan Silva et al (2015), plantearon que representar significaba hacer presente una idea, algo o alguien, y Jovchelovitch (1998) en sus estudios apuntó que la expresión "representaciones sociales" fue mencionada por primera vez en 1961 por el rumano Serge Moscovici, quien negó la dicotomía existente entre lo individual y lo social, hasta entonces representadas respectivamente por la Psicología que se encargaba de explicar al sujeto en su individualidad y por la sociología con sus estudios sobre la sociedad, en su colectivo (Alexandre, 2004).

Hallar todos estos factores dentro de la representación social del medio ambiente conlleva a una nueva configuración del concepto del MA, en donde aquellos fenómenos que hace décadas eran tomados como externos y que incidían de manera negativa sobre él, hoy lo integran y definen como un conjunto de elementos que van más allá de lo natural y trascienden hacia lo cultural, social y todo aquello que hace parte del contexto en donde se desenvuelve el ser humano y en el que él puede actuar para producir cambios. Así, se entiende que Pérez, Porras y Guzmán (2013), propongan que, al reconocer las representaciones sociales en la educación ambiental, se puede constituir en un referente para diseñar estrategias que permitan aportar a las prácticas, currículos y demás procesos pedagógicos, de los diferentes programas. Esto se ratifica en la Política Nacional de Educación Ambiental, de los ministerios de Educación Nacional y del Medio Ambiente (2002), al establecer como prioridad que la universidad desarrolle estrategias tendientes a introducir la pedagogía, la didáctica y la investigación en educación ambiental como componentes importantes de los diferentes programas de formación (Flores, 2012).

\section{Referencias}

Alexandre, M. (2004). Representação social: uma genealogia do conceito. Comum, 10(23), 122-38.

Babbie, E. (2000). Fundamentos de la Investigación Social. Buenos Aires: Thomson Editores.

Berger, P. L. \& Luckmann, T. (1988). La construcción social de la realidad. Barcelona: Herder (Edición original en inglés, 1966).

Capalbo, L. (2005). El Consumo, factor de la crisis ambiental. Ecoportal.net. Recuperado el 01 de diciembre de 2009 de http://www.ecoportal. net/content/view/full/43504

Ceirano, V. (2000). Las representaciones sociales de la pobreza. Revista Electrónica de Epistemología de Ciencias Sociales Cinta De Moebio, 9.

Crespo, J., \& Garcés, P. (2002). Guía de Trabajo en el Aula. Cuidar nuestro entorno es cuidar vida. Recuperado el 01 de diciembre de 2009 de http://www.ayudaenaccion.org/contenidos/ documentos/previo/cuidarnuestroentorn oescuidarvida.pdf

Durkheim, E. (1996). Reglas del Método Sociológico. Madrid: Orbis. 
Duveen, G. (2013). Prefácio. En S. Moscovici (Ed.), Representações sociais: Investigações em psicologia social. Petrópolis, RJ: Vozes.

Farah, A. (2011). Attitude and Social Representation. Procedia - Social and Behavioral Sciences, 30(2011), 1593-1597. DOI: 10.1016/j.sbspro. 2011.10.309

Ferreira, R. (2002). Representaciones sociales de medio ambiente y educación ambiental de docentes universitarios(as). Tópicos de Educación Ambiental, 4(10), 22-36.

Flores, R. (2008). Representaciones sociales del medio ambiente. Perfiles Educativos, 30 (120), 33-62.

Flores, R. (2010). Medio ambiente y educación ambiental: representaciones sociales de los profesores en formación. Magis, Revista Internacional de Investigación en Educación, 2(4), 401-414.

Flores, R. (2012). Investigación en educación ambiental. Revista Mexicana de Investigación Educativa, 17(55), 1019-1033.

Flores, R. (2013). Investigaciones de las representaciones sociales del medio ambiente en Brasil y México. Revista Actualidades Investigativas en Educación, 13(1), 1-20.

Gómez, M., Chamorro, G., Obispo, K., Parra, Y., Paba, C. \& Rodríguez, U. (2013). Representación social del psicólogo en el área de la salud: Un estudio cualitativo en la Facultad de Ciencias de la Salud de la Universidad del Magdalena. Psicología desde el Caribe, 30(1), 91-122.

González, M. (1998). La educación ambiental y formación del profesorado. Revista Iberoamericana de Educación, 16, 13-22. DOI: 10.35362/rie1601109

Jodelet, D. (1984). La representación social: fenómeno, concepto y teoría. En S. Moscovici (Ed.). Psicología Social II, Pensamiento y Vida Social, Psicología Social y Problemas Sociales (pp. 469506), Buenos Aires: Paidos.
Jodelet, D. (2011). Conferência de Denise Jodelet por ocasião do recebimento do título de doutor Honoris Causa da Universidade Federal do Rio de Janeiro. En A. J. Mazzotti (Ed.), Representações sociais: aspectos teóricos e aplicações à educação. Múltiplas Leituras, 18-43.

Jodelet, D. (2015). Problématiques psychosociales de l'approche de la notion de sujet. Cadernos de Pesquisa, 45(156), 315-327. DOI: 10.1590/198053143203

Jovchelovitch, S. (1998). Representações Sociais: para uma fenomenologia dos saberes sociais. Psicologia e Sociedade, 10(1), 54-68.

Knight, S. (2013). Creating a supportive environment for classroom dialogue. En S. Hennessy, P. Warwick, L. Brown, D. Rawlins \& C. Neale (Eds.), Developing interactive teaching and learning using the IWB. Open University Press.

Knight, S., Arastoopour, G., Williamson, D., Buckingham, S., \& Littleton, K. (2014). Epistemic networks for epistemic commitments. In International conference of the learning sciences. Boulder, CO: International Society of the Learning Sciences. Retrieved from http://oro.open.ac.uk/39254/.

Knight, S. \& Littleton, K. (2015). Thinking, interthinking, and technological tools. In R. Wegerif, L. Li, \& J. C. Kaufman (Eds.), The Routledge international handbook of research on teaching thinking (p. Section 7(al)). Routledge.

Lahitte, H., Hurrel, J., \& Malpartida, A. (1996). Relaciones 2: Crítica y expansión de la Ecología de las Ideas. Buenos Aires: Nuevo Siglo.

Laría, A. (2007). La crisis Ambiental cambiará la Conducta humana. Recuperado el 01 de diciembre de 2009 de http://www.perfil.com/ contenidos/2007/01/02/noticia_0057.html

Machado, W. (1996). Modelo didáctico para la interpretación ambiental en el Parque Nacional Laguna de La Restinga. Estado Nueva Esparta. (Tesis de Maestría) Universidad Pedagógica 
Experimental Libertador, Instituto Pedagógico de Caracas, Venezuela.

Moscovici, S. (1981). L'âge des foules. Paris: Fayard

Moscovici, S. (1984). La psychologie sociale. Paris: Presses Universitaires De France (PUF).

Moscovici, S. (1990). The Origin of Social Representations: A Response to Michael. Neu'ldeas in Phychology, 8(3), 383-388.

Moscovici, S. (1998). The history and actuality of social representations. In F. Uwe, The psychology of the social. New York: Cambridge University Press.

Moscovici, S. (2013). Representações sociais: Investigações em psicologia social. Petrópolis: Vozes.

Ministerio del Medio Ambiente. (2002). Política Nacional de Educación Ambiental. Bogotá: Ministerio del Medio Ambiente y Ministerio de Educación Nacional.

Navarro, O. (2004). Representación Social del agua y de sus usos. Psicología desde el Caribe, $14,222-236$.

Navarro, O. (2005). Psicología Ambiental: visión crítica de una disciplina desconocida. Revista Duazary, 2(1), 65-68.

Navarro, O. (2006). Representación social del agua. Pre-til, 10(4), 72-97.

Navarro, O. \& Gaviria, N. (2010). Representaciones sociales del habitante de la calle. Universitas Psychologica, 9(2), 345-355.

Navarro, O. (2013). Representación social del medio ambiente y de la contaminación del aire: efecto de imbricación de dos objetos. Revista CES Psicología, 6(1), 104-121.

Palavecinos, S., Martín, R., Díaz, M. J., Piñeiro, C., Benayas, J., ... Jiménez, A. (2008). Comportamiento proambiental: Estudio del comportamiento de queja ciudadana.
Implementación de un sistema participativo de gestión ambiental en Madrid. Revista de Psicología Social, 23(2), 243-257.

Pérez, M., Porras, Y., \& Guzmán, H. (2013). Representaciones sociales de la educación ambiental y del campus universitario. Una mirada de los docentes en formación de la Universidad Pedagógica Nacional. TED. 34 (julio-diciembre) 47-69.

Real Academia de la Lengua Española. (2004), El Pequeño Larousse Multimedia.

Reigota, M. (1995). Meio Ambiente e Representação Social. São Paulo: Cortez.

Rodríguez, L., \& López, E. (2005). Representaciones sociales de conservación ambiental en la comunidad la flor de Marqués de Comillas, Chiapas. Horizonte Sanitario, 4(2), 1-12.

Rodríguez, U. (2006). El Impacto del Hombre en la Naturaleza: Una Perspectiva desde la Psicología Ambiental y la Economía. Revista Duazary, 3(1), 60-63.

Rodríguez, U. (2009). Representación socio-espacial del Centro Histórico de la ciudad de santa Marta. Aproximación al constructo de identidad de lugar. Duazary, 6(2), 102-111.

Rodríguez, U. (2011). Representación social y análisis del Referido semántico del medio ambiente y la educación ambiental en la Universidad del Magdalena. Duazary, 8(1), 24-33.

Rodríguez, U., Cortés, S., \& Varela, M. (2007). Mapas Mentales del Centro Histórico de Santa Marta. Recuperado el 25 de Marzo de 2007, de: http://www.psicologiacientifica.com/bv/ psicologia-231-6-mapas-mentales-del-centrohistorico-de-santa-marta.html.

Rodríguez, U., Espitia, J., Montenegro, W., Ortiz, F., \& Suárez, Y. (2013). Representación social de la memoria en una muestra universitaria de ingeniería de sistemas y antropología. Duazary, 11(1), 7-13. 
Rodríguez, U. \& Paba, C. (2013). Representación Social de la Crisis Ambiental. Psicogente, 16(29), 84-102.

Ruíz J. I. \& Coy A. (2004). Esquemas cognitivos de base, contenido semántico y estructura de las representaciones sociales de la democracia. Acta Colombiana de Psicología, 12, 5-17

Schutz,A. \& Luckmann,T. (1977). Las estructuras del mundo de la vida. Buenos Aires: Amorrortu.

Silva, C., Carmo, G., \& Silva, A. (2015). Breves observações sobre a teoria das representações sociais de Serge Moscovici e a interdisciplinaridade. Estudos Interdisciplinares em Psicologia, 6(2), 59-70. DOI: 10.5433/2236-6407.2015v6n2p59
Suárez, N. \& Márquez, G. (2004). Estado del arte de la investigación sobre medio ambiente en el Caribe Colombiano. Cartagena de Indias: Cartagena: Observatorio del Caribe.

Tilbury, D. (2004). Reconceptualizando la educación ambiental para un Nuevo Siglo. Tópicos en Educación Ambiental, 3(7), 65-73.

Torres, M. (1998). La educación ambiental: una estrategia flexible, un proceso y unos propósitos en permanente construcción. La experiencia de Colombia. Revista Iberoamericana de Educación, 16, 23-27.

Touraine, A. (2007). Penser autrement. Paris: Fayard. 